\title{
Research on Fault Diagnosis of Hydraulic System of Fast Erecting Device Based on Fuzzy Neural Network
}

\author{
Yangbing Zheng ${ }^{1,2}$, Xiao Xue ${ }^{3, *}$ and Jisong Zhang ${ }^{2}$ \\ ${ }^{1}$ College of Mechanical and Electronic Engineering, Nanyang Normal University, \\ Nanyang 473061, Henan, China \\ ${ }^{2}$ Qinghai Wandong Ecological Environment Development Co.LTD, Geermu \\ 816000, Qinghai, China \\ ${ }^{3}$ School of Information Engineering, Nanyang Institute of Technology, Nanyang \\ 473004, Henan, China \\ E-mail:x909@163.com \\ ${ }^{*}$ Corresponding Author
}

Received 12 October 2021; Accepted 25 October 2021;

Publication 07 January 2022

\begin{abstract}
In order to improve the fault diagnosis effectiveness of hydraulic system in erecting devices, the fuzzy neural neural network is applied to carry out fault diagnosis of hydraulic system. Firstly, the main faults of hydraulic system of erecting mechanism are summarized. The main faults of hydraulic system of erecting devices concludes abnormal noise, high temperature of hydraulic oil of hydraulic system, leakage of hydraulic system, low operating speed of hydraulic system, and the characteristics of different faults are analyzed. Secondly, basic theory of fuzzy neural network is studied, and the framework of fuzzy neural network is designed. The inputting layer, fuzzy layer, fuzzy relation layer, relationship layer after fuzzy operation and outputting layer of fuzzy neural network are designed, and the corresponding mathematical models are confirmed. The analysis procedure of fuzzy neural network is established. Thirdly, simulation analysis is carried out for a hydraulic system
\end{abstract}

International Journal of Fluid Power, Vol. 23_2, 141-160.

doi: 10.13052/ijfp1439-9776.2321

(C) 2022 River Publishers 
in erecting device, the BP neural network reaches convergence after 600 times iterations, and the fuzzy neural network reaches convergence after 400 times iterations, fuzzy neural network can obtain higher accuracy than BP neural network, and running time of fuzzy neural network is less than that of BP neural network, therefore, simulation results show that the fuzzy neural network can effectively improve the fault diagnosis efficiency and precision. Therefore, the fuzzy neural network is reliable for fault diagnosis of hydraulic system in erecting devices, which has higher fault diagnosis effect, which can provide the theory basis for healthy detection of hydraulic system in erecting devices.

Keywords: Fault diagnosis, hydraulic system, erecting device, fuzzy neural network.

\section{Introduction}

With rapid development of Chinese social economy, hydraulic system of fast erecting devices has a rapid development. The fast erecting devices have been applied in various lifting operations and safety projects, and can effectively improve economic interests of China, and reduce capital investment cost. However, there are many safety faults in the application process due to relatively use of erecting devices and influence of various factors. In addition, the professional quality of hydraulic system repair staff is poor, therefore many problems exist in the repair process of hydraulic system of fast erecting device. It is easy to break down in running process, which has a very adverse impact on improving the working efficiency of fast erecting device. When the erecting device works, it can obtain the power with help of the hydraulic system, the hydraulic system has the function of stepless speed regulation, which can achieve a variety of separate actions. When is is not fully loaded, each executive element in series can be controlled to complete the action respectively. In the operation process, it is usually completed by controlling hydraulic pump, oil pipe, oil tank and other components [1].

The faults of the hydraulic system of erecting device mainly comes from many aspects, and the reasons can be divided into five kinds, firstly quality of component in the erecting device circuit is poor, or multiple circuits interfere with each other. At same time, it is also possible to have the failure due to abnormal conditions when a erecting device circuit element operates alone. When erecting device circuit component fails, the possibility of fault of hydraulic cylinder is greatest. Secondly, during actual operation 
of erecting devices, there will be certain safety faults in the hydraulic system because the working medium is not scientifically and reasonably selected, or the erecting device is not well managed. According to a large number of investigations, about $70 \%-85 \%$ of faults of hydraulic system are caused by contaminated objects in the hydraulic oil. Thirdly in security commissioning of hydraulic system of erecting device, there are operation errors or unreasonable operation. In this case, the safety faults of the hydraulic system will occur. Fourthly, there are errors in the operation mode of erecting device in the process of practical application, such as not turning off the emergency switch in time, which directly lead to the failure of the hydraulic system of erecting device. Finally, during the actual operation of erecting device, there is security faults of hydraulic system because of high wind force under the working environment or the impact of foreign objects [2].

The characteristics signal of hydraulic system fault is often disturbed by random noise, which makes the signal-to-noise ratio of signal is very low. Especially when the system has early fault, its weak fault information is completely submerged in the noise, which brings the great difficulties to the feature extraction and fault classification of fault signal. How to suppress the interference of noise, improve the signal-to-noise ratio is a key problem in the fault diagnosis of hydraulic system of erecting devices. In the real measured fault characteristic signal, there is only Gaussian whitening noise, but also Gaussian colored noise with unknown spectral structure. The traditional method can not effectively deal with these signals. The high order statistics method proposed in recent years can effectively cope with these noises. The structural knowledge expression ability of fuzzy neural network is very good, which can better describe the qualitative knowledge of the system, but also solve the uncertain knowledge, it has very good nonlinear mapping ability good adaptive and self-learning ability, and strong fault tolerance ability. It can be effectively applied to the fault diagnosis of complex systems. This research applies the fuzzy neural network to carry out fault diagnosis of hydraulic system of erecting devices [3]. The main contribution of this research is put forward a novel fault diagnosis of hydraulic system of fast erecting device based on fuzzy neural network.

\section{Normal Faults of Hydraulic System of Erecting Mechanism}

The common faults of hydraulic system of erecting devices concludes the following aspects. 


\subsection{Abnormal Noise}

The hydraulic pump of erecting device is main noise source, there noises are harsh, will affect the normal use of hydraulic system, and reduce the life of hydraulic system leading to paralysis of the whole erecting devices. The reasons of causing abnormal noise are as follows: the oil tank respirator and oil inlet pipeline are blocked, the oil pump is empty, the hydraulic oil is unqualified, and the mechanical faults [4].

\subsection{High Temperature of Hydraulic Oil of Hydraulic System in Erecting Devices}

The hydraulic oil is the working medium of hydraulic system in erecting devices, the pressure potential energy generated by hydraulic oil can transmit the power. All energy loss of hydraulic system is converted into heat energy that is transmitted to hydraulic oil and hydraulic components, and then the temperature of hydraulic oil will increase. When the working temperature ranges from $40^{\circ} \mathrm{C}$ to $70^{\circ} \mathrm{C}$, the hydraulic system of erecting devices can obtain the good working performance [5].

If the hydraulic oil temperature is too high, the performance of hydraulic system of erecting devices will be reduced. And many adverse phenomena will occur. For example, the hydraulic system produces large leakage, the performance of hydraulic system is reduced, and the viscosity of hydraulic oil is reduced. The wear between components of erecting devices increases, and the hydraulic oil film is damaged, and then the temperature of hydraulic oil increases. The hydraulic oil is oxidized, the service life is shortened, and the formed colloid is deposited on the surface of hydraulic components, the small holes of hydraulic system of erecting devices is blocked. The sealing structure of hydraulic system in erecting devices fails, the pressure loss increases, the controlling valve fails and the mechanical loss increase [6].

\subsection{Leakage of Hydraulic System in Erecting Devices}

The leakage of hydraulic system in erecting devices mainly concludes internal and external leakage. The internal leakage mainly refers to the internal leakage from hydraulic components to low pressure chamber, the external leakage refers to the outward leakage of oil from hydraulic components. The leakage of hydraulic system will affect the performance of whole system, and lead to deceasing of pressure, the working process of erecting devices is not stable, and the oil pump of hydraulic system is easy to be damaged. The main reason of this fault is damage of the seal components of erecting devices [7]. 


\subsection{Low Operating Speed of Hydraulic System in Erecting Devices}

The main reason of low operating speed of hydraulic system has many aspects: failure of the controlling valve, fault of oil supplying system, and the fault of hydraulic cylinder.

\section{Basic Theory of Fuzzy Neural Network}

The $k$ th order moment and $k$ th order cumulative of random variable $x$ is defined by [8]

$$
\begin{aligned}
M_{k} & =\int_{-\infty}^{+\infty} x^{k} f(x) d x \\
C_{k} & =(-j)^{k} \Psi^{k}(0)
\end{aligned}
$$

where $f(x)$ denotes the probability density function of random variable $x, \Psi(\omega)$ denotes the second characteristic function, if $x$ serves Gaussian distribution $N\left(0, \sigma^{2}\right)$, and the following conditions can be obtained:

$$
C_{1}=0, C_{2}=\sigma^{2}, C_{k} \equiv 0(k \geq 3)
$$

$x(n)$ is the stable random process, the second moment and the third moment are defined by [9]

$$
\begin{gathered}
m_{2 x}\left(\tau_{1}\right)=E\left[x(n) x\left(n+\tau_{1}\right)\right] \\
m_{3 x}\left(\tau_{1}, \tau_{2}\right)=E\left[x(n) x\left(n+\tau_{1}\right) x\left(n+\tau_{2}\right)\right]
\end{gathered}
$$

Suppose random variables $\left\{x_{i}\right\}$ and $\left\{x_{j}\right\}$ are independent of each other, let $[10]$

$$
\vec{Z}=\left[x_{1}+y_{1}, \ldots, x_{k}+y_{k}\right]=\vec{X}+\vec{Y}
$$

And then the following equation can be obtained:

$$
\Psi(\omega)=\Psi_{x}(\omega)+\Psi_{y}(\omega)
$$

where $\Psi_{x}(\omega)$ and $\Psi_{y}(\omega)$ are second characteristic functions of random variables $\left\{x_{i}\right\}$ and $\left\{x_{j}\right\}, \Psi(\omega)$ are the second characteristics function of sum between random variables $\left\{x_{i}\right\}$ and $\left\{x_{j}\right\}$. When the signal contains additive Gaussian colored noise, in theory, the high order cumulant can completely suppress the effect of noise, so as to improve the signal to noise ratio. 
Neural network basically belongs to the underlying computing structure and algorithm, extract information from the trained system, and the fuzzy logic usually processes oral and language information obtained from experts at a high level than neural network, but fuzzy logic does not have the learning function. At same time, because the internal layers of neural network are fuzzy to users. The mapping rules of network are invisible. So there is a theory of integrating fuzzy logic and neural network into a system, which makes it have both advantages. For example, fuzzy logic can have the learning function, optimization function and linked function of neural network, so that the low level learning and calculation functions of the neural network can be introduced into fuzzy system, and the high level fuzzy reasoning function of fuzzy system can also be introduced into neural network. For the neural network, the neural network can make the neural network more and more transparent, by possibly explaining the weights after learning stage or prior structuring the neural network. Therefore, if the neural network and fuzzy system is combined to establish fuzzy neural network, which is used to classify the faults of hydraulic system, and the advantages of fuzzy system and neural network can be gave full play to improve the accuracy and efficiency of fault diagnosis results [11].

There are many uncertain factors affecting the fault of hydraulic system in erecting devices, which can not be quantified, including human logical thinking. Therefore, a definite numerical method is needed to analyze and judge the fuzzy factors. When using classical mathematics to analyze fuzzy factors, it is difficult to obtain high-precision estimation results. Fuzzy mathematics can better realize the quantification of model problems when dealing with fuzzy problems. At the same time, computer technology has made great progress, which provides a better intelligent method for dealing with fuzzy problems. In 1965, the American scholar Chad first put forward the concept of fuzzy set, and determined the middle difference by introducing the concept of membership, thus forming the theory of fuzzy mathematics. Fuzzy mathematics is a new theory based on fuzzy problems. The main connotation of the fuzziness of the influencing factors of hydraulic system faults has no clear boundary. It is a gray problem of the differences between various factors. Fuzzy mathematics can effectively deal with the problem of uncertainty [12].

The basic theory of fuzzy mathematical is to build a mathematical model for representing fuzzy problems based on fuzzy sets, fuzzy mathematical further improves the concept of sets. By specifying the corresponding operation rules and transformation methods, and by expanding the basis of fuzzy 
mathematics, the fuzzy system model can be built based on quantitative method. In traditional mathematical theory, only 0 and 1 represents the logic relationship, the fuzzy mathematics can improve the traditional mathematics, quantifies the fuzzy relationship, and expresses the membership degree through number between 0 and 1 , so as to deal with the fuzzy problem more effectively [13].

In the process of hydraulic system faults diagnosis, there are many fuzzy factors, and the logic correlation should be applied to establish a fuzzy mathematical model. The numerical simulation of fuzzy mathematical model can be realized by computer program, The quantification of fuzzy factors is to describe them mathematically by using fuzzy mathematical methods. For example, if the membership degree of a factor is set to 1 , the similar factors or completely different factors can be expressed by using membership degree between 0 and 1 [14].

Fuzzy mathematics can effectively deal with problems related to uncertain problems, fuzzy sets can be used to express fuzzy problems with uncertain boundaries. Fuzzy theory can obtain the fuzzy object in the fault diagnosis of hydraulic system of erecting devices, and construct the corresponding fuzzy mathematical model.

The set $A$ is known, a variable $x$ exists in the space,there are two cases, $x \in A$ or $x \notin A$, this problem can be described by

$$
A(x)= \begin{cases}0 & x \notin A \\ 1 & x \in A\end{cases}
$$

where, $A(x)$ represents the eigenvalue of $A$, extends the eigenfunction to the fuzzy set, and extends the set to set taking 0 and 1 to fuzzy set, and the interval of fuzzy set is $[0,1]$.

Definition 1: the global domain $U$ is known, when function takes the fuzzy number 0 and 1 in the global domain, that is $A$.

Definition 2: when the fuzzy number $A$ is any fuzzy number in the whole domain $U$, there is a variable $\lambda \in[0,1]$, and there is the following set [15]:

$$
U \lambda=\{x \mid x \in U, A(x) \geq \lambda\}
$$

Then, $U \lambda$ is the $\lambda$ cut set on the whole domain $U, \lambda$ is the confidence level.

$U \lambda$ is only an ordinary set, because the fuzzy set lacks clear boundaries, the corresponding membership degree can be determined by selecting different sets, so as to obtain the fuzzy mathematical model. Cut sets can be used to 
realize the transformation from fuzzy sets to ordinary sets. Fuzzy set is a set with movable boundaries, which is inversely proportional to the confidence level.

Definition 3: if there are two fuzzy sets $A$ and $B$ in global domain, the sum set, the intersection set and coset of the two fuzzy sets also belongs to fuzzy sets. The operation of fuzzy sets can be applied in any number of fuzzy operations .

Definition 4: a matrix is known, which is between 0 and 1 , it is named as fuzzy matrix. The fuzzy identity matrix is defined by $I$. The fuzzy zero matrix locates 0 , and the matrix with all elements of 1 is defined as $J$.

Definition 5: given two fuzzy matrices $A$ and $B$. the product of the two fuzzy matrices are also fuzzy matrices. In fuzzy mathematical theory, there are two important concepts which are membership degree and closeness degree [16].

Membership degree: there is a element $x$ in global domain $U$, and there is also a corresponding function $A(x)$, then $A$ is named as fuzzy set. $A(x)$ is the membership degree of element $x$ to fuzzy set $A$. The membership degree can be used to describe the degree of element $x$ belonging to fuzzy set. The membership degree function ranges from 0 to 1 . There are many methods of selecting membership degree, such as example method, fuzzy statistics method and ranking method. The membership degree can be used to reach fuzzy control, the fuzzy control can be effectively achieved through correctly selecting membership function, and the effectiveness of applying fuzzy mathematics to cope with uncertain problem. Therefore the fuzzy membership function plays an important role in fuzzy mathematics. In fuzzy set, the eigen value is the any number between 0 and 1 . In generally, the expert score method, statistical method and experience method can be used to confirm the membership degree function. Therefore, the intelligent method is an effective method to amend the subjective method. In recent years, there is no effective method of confirming the membership function, when the different membership functions are used to describe the united fuzzy factors, and different results may be obtained in calculation process. The membership function is not unchanged, and the membership degree function can be amended according to the real situation of hydraulic system of erecting devices, and the fault diagnosis precision can be improved.

Closeness degree: this index can describe the close degree between two fuzzy sets, two fuzzy sets are given, the internal product between the two sets are 
calculated by [17]

$$
A \cdot B=\widehat{X e U}_{\widehat{A}}(A(x) \vee B(x))
$$

The external product of the two fuzzy sets is calculated by

$$
A \otimes B=\underset{X e U}{\vee}(A(x) \wedge B(x))
$$

When the fuzzy set $B$ constantly closes t fuzzy set $A$, the internal product increases, and the external product decreases. When the closeness degree between fuzzy set is not described only by internal product and external product, the closeness between fuzzy sets should be denoted through comprehensively utilizing internal product and external product [18].

The fuzzy system and neural network are combined to establish the fuzzy neural network, the fuzzy neural network has good knowledge expression level and fault tolerance ability and can express and store knowledge well. The fuzzy neural network is applied in fault diagnosis of hydraulic system in erecting devices, the fuzzy membership degree can be used to describe the changing trend of affecting factors of fault diagnosis of hydraulic system in erecting devices, the neural element and connection weight are used to describe the fault types of hydraulic system in erecting devices, and then the correct knowledge can be obtained, the relating knowledge can be stored easily, and the fault diagnosis accuracy of hydraulic system in erecting devices can be improved [19].

The fuzzy neural network concludes five layers concluding inputting layer, fuzzy layer, fuzzy relation layer, relationship layer after fuzzy operation, outputting layer, the structure of fuzzy neural network is shown in Figure 1.

Inputting layer: this layer can transfer the inputting variables to the element of next layer, the inputting vector is expressed by [20]

$$
x=\left[x_{1}, x_{2}, \ldots, x_{n}\right]^{T}
$$

The corresponding membership degree function is expressed by

$$
\alpha_{j}(x)=\varphi_{j}\left(\frac{\left\|x-c_{i j}\right\|}{\sigma_{i j}}\right)=e^{-\frac{\left\|x-c_{i j}\right\|^{2}}{\sigma_{i j}^{2}}}, \quad j=1,2, \ldots, \sum_{i=1}^{n} N_{i}
$$

where $c_{i j}$ and $\sigma_{i j}$ are used to denote center and width of membership degree function, $N_{i}$ denotes the dimensional degree of $i$ th inputting variable. 


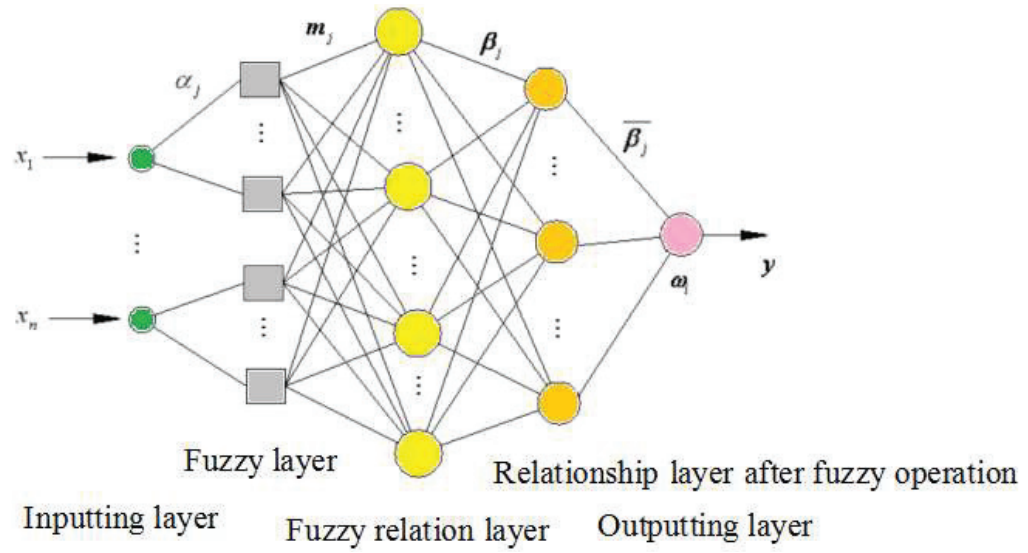

Figure 1 Structural diagram of fuzzy neural network.

Fuzzy layer: every node in this layer corresponds to a language variable, which can be applied to solve the input of membership degree degree, and the membership degree function is expressed by

$$
m_{j}=e^{\frac{x-c_{i j}}{\sigma_{i j}^{2}}}, \quad j=1,2, \ldots, \sum_{i=1}^{n} N_{i}
$$

Fuzzy relation layer, this layer can carry out fuzzy sum of inputting variables of fuzzy layer and can obtain the fuzzy relationship vector, and the corresponding expression is listed as follows:

$$
\beta_{j}=\min \left\{m_{1}, m_{2}, \ldots, m_{k}\right\}, \quad j=1,2, \ldots, N_{A}, \quad k=1,2, \ldots, N_{n}
$$

where $N_{A}=\prod_{i=1}^{n} N_{i}$.

Relationship layer after fuzzy operation: this layer can carry ou normalized calculation of relationship vectors, and the calculation expression is listed as follows:

$$
\begin{gathered}
\overline{\beta_{j}}=\frac{\beta_{j}}{\sum_{i=1}^{N_{A}} \beta_{i}}, \quad j=1,2, \ldots, N_{A} \\
\sum_{i=1}^{N_{A}} \overline{\beta_{j}}=1
\end{gathered}
$$


Outputting layer: this layer can obtain the weighted linear sum of relationship strength, and the corresponding expression is listed as follows:

$$
y=\sum_{i=1}^{N_{A}} \omega_{i} \bar{\alpha}_{i}=\omega^{T} \Lambda
$$

where, $\omega_{i}$ represents the connection weight between nodes, $\omega$ represents connection vector between nodes,

$$
\omega=\left[\omega_{1}, \omega_{2}, \ldots, \omega_{N_{A}}\right]^{T}, \quad \Lambda=\left[\overline{\alpha_{1}}, \overline{\alpha_{2}}, \ldots, \overline{\alpha_{N_{A}}}\right]^{T} .
$$

The fault diagnosis procedure of hydraulic system in erecting devices is listed as follows:

Step 1: Sample data standardization

The input hydraulic system fault characteristic data of erecting devices are normalized and grouped. One group of data is used as training data and the other group of data is used as test data.

Step 2: network initialization.

The classification data and weight matrix are initialized.

Step 3: Fuzzy neural network training.

The standardized training data set is imported into fuzzy neural network for training, and the classification results are output.

Step 4: training is end.

In the process of training, constantly detect whether the training meets the end conditions. If satisfied, the training ends and the network model is output.

Step 5: data test.

The test data set is imported into the trained network model to test the fault diagnosis ability of the network.

\section{Fault Diagnosis Analysis of Hydraulic System in Erecting Devices}

In the fault diagnosis of hydraulic system in erecting devices, the original data collected, that is, the input of fuzzy neural network, mainly include the following indexes: system operation noise, hydraulic oil temperature, working speed of hydraulic cylinder and leakage of hydraulic system. The above four 
performance indexes can reflect the operation status of the hydraulic system of erecting devices. Therefore, the above four performance indexes are used as the input of the fuzzy neural network, that is, the fuzzy neural network includes four input nodes.

Through signal filtering, weighted calculation and statistical analysis, the results obtained from these operations can obtain the fault symptom signals of automobile hoist hydraulic system, such as temperature deviation, working speed deviation, noise deviation, etc. The common faults of the hydraulic system of the automobile hoist are: the oil tank respirator and the oil inlet pipeline are blocked, the oil pump is empty, the control valve is out of order, the oil supply system is out of order, and the hydraulic cylinder is out of order. In addition, there are 6 output nodes of the fuzzy neural network.

Firstly, high-order statistics method is used to extract fault features to suppress the influence of background noise on diagnosis results. Secondly, through signal filtering, weighted calculation and statistical analysis, the results obtained from these operations can acquire the fault symptom signals of hydraulic system in erecting devices, such as temperature deviation, working speed deviation, noise deviation, etc. The common faults of the hydraulic system of the automobile hoist are: the oil tank respirator and the oil inlet pipeline are blocked, the oil pump is empty, the control valve is out of order, the oil supply system is out of order, and the hydraulic cylinder is out of order. In addition, there are six output nodes of the fuzzy neural network.

Relationship between fault symptoms and causes of hydraulic system in erecting devices is listed in Table 1.

In order to verify the effectiveness of the proposed method, the BP neural network is also used to carry out fault diagnosis analysis of hydraulic system in erecting devices. And the error iteration curve is shown in Figure 2. As seen from Figure 2, the BP neural network reaches convergence after 600 times iterations, and the fuzzy neural network reaches convergence after 400 times iterations, therefore the fuzzy neural network has quicker convergence speed.

The part fault diagnosis results of hydraulic system of erecting devices based on fuzzy neural network are listed in Table 2, as seen from Table 2, the fault point approaches to 1 , and the non fault point approaches to 2 , the fault diagnosis results are consistent with the real situation of hydraulic system in erecting devices.

The fuzzy neural network is applied to carried out fault diagnosis of hydraulic system in erecting devices, and the results are listed in Table 3. 
Table 1 Relationship between fault symptoms and causes of hydraulic system in erecting devices

\begin{tabular}{llcccccc}
\hline \multirow{2}{*}{ Input nodes } & 1 & 2 & 3 & 4 & 5 & 6 \\
& $\begin{array}{l}\text { Training Samples } \\
\text { Operating noise of hydraulic system in }\end{array}$ & 0 & 1 & 0.2 & 0.6 & 0.2 & 1 \\
& $\begin{array}{l}\text { erecting devices } \\
\text { Temperature of hydraulic oil }\end{array}$ & 0 & 1 & 0.7 & 1 & 0.3 & 0 \\
& Working rotating speed of hydraulic & 0 & 1 & 0.7 & 0.3 & 0.7 & 0.5 \\
& cylinder & & & & & & \\
& Leakage of hydraulic system & 0 & 1 & 0.3 & 0.6 & 1 & 0 \\
Output nodes & 0 & 0 & 0 & 1 & 0 & 0 \\
& Blocking of oil inlet pipeline & 0 & 0 & 0 & 0 & 1 & 0 \\
& Oil pump suction & 0 & 0 & 0 & 0 & 0 & 1 \\
& Failure of controlling valve & 0 & 0 & 0 & 1 & 0 & 0 \\
& Fault of oil supply system & 1 & 0 & 0 & 0 & 0 & 0 \\
& Fault of hydraulic cylinder & 0 & 1 & 0 & 0 & 0 & 0 \\
\hline & No fault & & & & & & \\
\hline
\end{tabular}

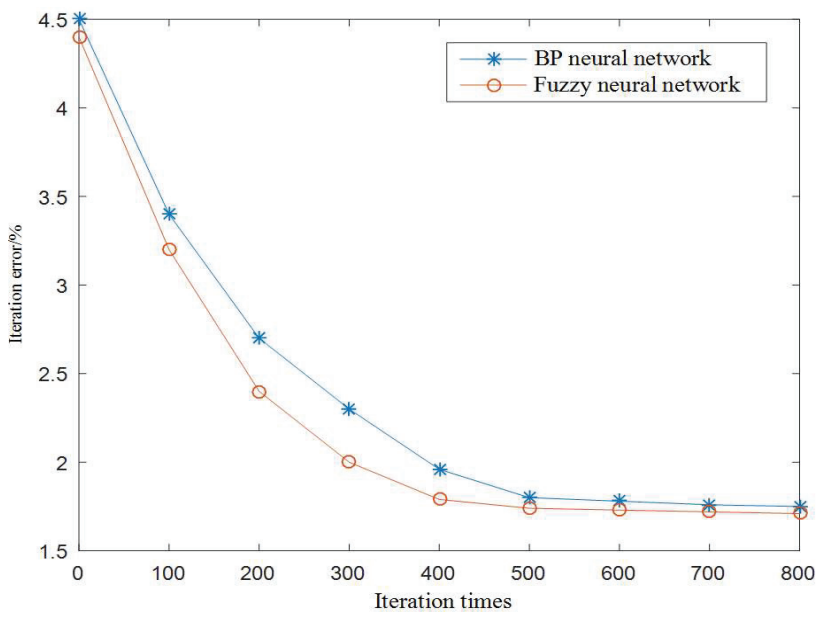

Figure 2 Error iteration curves of the two methods.

As seen from Table 3, the fuzzy neural network can obtain the higher fault diagnosis accuracy than BP neural network for hydraulic system in erecting devices. Therefore the fuzzy neural network has higher fault diagnosis precision of hydraulic system. The analysis results show that the fuzzy neural network is reliable for fault diagnosis of hydraulic system in erecting devices, which has higher fault diagnosis effect, which can provide the theory basis for healthy detection of hydraulic system in erecting devices. 
Table 2 Part fault diagnosis results of hydraulic system of erecting device

\begin{tabular}{lccccccl}
\hline No & \multicolumn{7}{c}{ Output Results } \\
\hline 1 & 0.022 & 0.004 & 0.017 & 0.982 & 0.043 & 0.007 & Blocking of oil inlet pipeline \\
2 & 0.033 & 0.026 & 0.11 & 0.052 & 0.993 & 0.039 & Oil pump suction \\
3 & 0.004 & 0.001 & 0.045 & 0.006 & 0.032 & 0.988 & Failure of controlling valve \\
4 & 0.0026 & 0.007 & 0.003 & 0.993 & 0.046 & 0.028 & Fault of oil supply system \\
5 & 0.995 & 0.005 & 0.007 & 0.016 & 0.038 & 0.052 & Fault of hydraulic cylinder \\
6 & 0.026 & 0.996 & 0.027 & 0.006 & 0.051 & 0.063 & No fault \\
7 & 0.044 & 0.062 & 0.044 & 0.979 & 0.005 & 0.025 & Blocking of oil inlet pipeline \\
8 & 0.036 & 0.022 & 0.018 & 0.985 & 0.006 & 0.026 & Fault of oil supply system \\
9 & 0.032 & 0.055 & 0.032 & 0.016 & 0.985 & 0.037 & Oil pump suction \\
10 & 0.017 & 0.969 & 0.021 & 0.006 & 0.023 & 0.018 & No fault \\
11 & 0.007 & 0.042 & 0.062 & 0.006 & 0.992 & 0.005 & Oil pump suction \\
12 & 0.005 & 0.017 & 0.026 & 0.022 & 0.016 & 0.984 & Failure of controlling valve \\
13 & 0.028 & 0.036 & 0.025 & 0.991 & 0.009 & 0.003 & Blocking of oil inlet pipeline \\
14 & 0.055 & 0.032 & 0.008 & 0.026 & 0.026 & 0.977 & Failure of controlling valve \\
15 & 0.016 & 0.033 & 0.043 & 0.015 & 0.005 & 0.996 & Failure of controlling valve \\
16 & 0.985 & 0.025 & 0.017 & 0.032 & 0.005 & 0.036 & Fault of hydraulic cylinder \\
17 & 0.036 & 0.026 & 0.019 & 0.015 & 0.984 & 0.005 & Oil pump suction \\
18 & 0.007 & 0.043 & 0.027 & 0.967 & 0.042 & 0.036 & Blocking of oil inlet pipeline \\
19 & 0.005 & 0.026 & 0.024 & 0.036 & 0.005 & 0.943 & Failure of controlling valve \\
20 & 0.006 & 0.005 & 0.035 & 0.021 & 0.029 & 0.975 & Failure of controlling valve \\
\hline & & & & & & &
\end{tabular}

Table 3 Fault diagnosis results of hydraulic system in erecting devices

\begin{tabular}{lccc}
\hline & Number & \multicolumn{2}{c}{ Fault Diagnosis Accuracy Rate } \\
\cline { 3 - 4 } Fault Type & of Samples & BP Neural Network & Fuzzy Neural Network \\
\hline Blocking of oil inlet pipeline & 20 & $97.2 \%$ & $99.5 \%$ \\
Oil pump suction & 31 & $97.7 \%$ & $100 \%$ \\
Failure of controlling valve & 27 & $96.3 \%$ & $98.9 \%$ \\
Fault of oil supply system & 32 & $98.3 \%$ & $100 \%$ \\
Fault of hydraulic cylinder & 26 & $97.5 \%$ & $98.7 \%$ \\
No fault & 30 & $98.5 \%$ & $100 \%$ \\
\hline
\end{tabular}

The running time of BP neural network and fuzzy neural network are obtained, which is listed in Table 4.

As seen from Table 4, the running time of fuzzy neural network is less than that of BP neural network, therefore the fuzzy neural network has higher fault diagnosis efficiency of hydraulic system of erecting devices. 
Table 4 Running time of different methods

\begin{tabular}{lcc}
\hline & \multicolumn{2}{c}{ Running Time/s } \\
\cline { 2 - 3 } No. & BP Neural Network & Fuzzy Neural Network \\
\hline 1 & 35.4 & 28.1 \\
2 & 33.1 & 27.4 \\
3 & 34.5 & 26.7 \\
4 & 29.3 & 25.8 \\
5 & 30.5 & 28.4 \\
6 & 32.8 & 28.9 \\
7 & 29.4 & 25.4 \\
8 & 30.7 & 25.1 \\
9 & 32.6 & 28.3 \\
10 & 31.6 & 27.6 \\
11 & 32.6 & 29.0 \\
12 & 31.4 & 27.7 \\
13 & 34.7 & 29.7 \\
14 & 33.6 & 29.6 \\
15 & 32.2 & 28.5 \\
16 & 31.6 & 28.4 \\
17 & 33.2 & 28.3 \\
18 & 35.6 & 31.0 \\
19 & 34.1 & 30.6 \\
20 & 32.8 & 28.5 \\
\hline
\end{tabular}

\section{Conclusions}

The fuzzy neural network is constructed for fault diagnosis of hydraulic system in erecting devices, and high order cumulant method is applied to improve the signal to noise ratio of fault characteristic signal, which can eliminate the effect of noise on diagnosis results. Simulation results show that the fuzzy neural network has obvious advantages on fault diagnosis of hydraulic system. The fault efficiency and precision of fuzzy neural network can be improved effectively.

\section{Acknowledgment}

This work is supported by the Henan Provincial Key Science and Technology Research Projects (NO. 202102210126) and University-level STP project. 


\section{References}

[1] Xiancheng Ji, Ya Ren, Hesheng Tang, Jiawei Xiang, DSmT-based threelayer method using multi-classifier to detect faults in hydraulic systems, Mechanical Systems and Signal Processing, 2021, 153(5):107513.

[2] Salman Ijaz, Lin Yan, Mirza Tariq Hamayun, Cun Shi, Active fault tolerant control scheme for aircraft with dissimilar redundant actuation system subject to hydraulic failure, Journal of the Franklin Institute, 2019, 356(3):1302-1332.

[3] Siavash Sharifi, AliTivaya S. Mehdi, Rezaeia Mohammad, Zareinej Bijan Mollaei-Dariani, Leakage fault detection in Electro-Hydraulic Servo Systems using a nonlinear representation learning approach, ISA Transactions, 2018, 73(2):154-164.

[4] Andrea Macaluso, Giovanni Jacazio, Prognostic and Health Management System for Fly-by-wire Electro-hydraulic Servo Actuators for Detection and Tracking of Actuator Faults, Procedia CIRP, 2017, 59(1):116-121.

[5] V. Indira, R. Vasanthakumari, R. Jegadeeshwaran, V. Sugumaran, Determination of minimum sample size for fault diagnosis of automobile hydraulic brake system using power analysis, Engineering Science and Technology, an International Journal. 2015, 18(1):59-69.

[6] R. Ogawa, T. Kido, T. Kido, T. Mochizuki, Effect of augmented datasets on deep convolutional neural networks applied to chest radiographs, Clinical Radiology, Vol. 74, No. 9, 2019, pp. 697-701.

[7] Ji-Yoon Kim, Sung-Bae Cho, Exploiting deep convolutional neural networks for a neural-based learning classifier system, Neurocomputing, Vol. 354, No. 8, 2019, pp. 61-70.

[8] Wenyi Huang, Junsheng Cheng, Yu Yang, Gaoyuan Guo, An improved deep convolutional neural network with multi-scale information for bearing fault diagnosis, Neurocomputing, Vol. 359, No. 9, 2019, pp. 7792.

[9] Tao Wan, Shusong Xu, Chen Sang, Yulan Jin, Zengchang Qin, Accurate segmentation of overlapping cells in cervical cytology with deep convolutional neural networks, Neurocomputing, Vol. 365, No. 11, 2019, pp. $157-170$.

[10] Dezun Zhao, Tianyang Wang, Fulei Chu, Deep convolutional neural network based planet bearing fault classification, Computers in Industry, Vol. 107, No. 5, 2019, pp. 59-66. 
[11] Boukaye Boubacar Traore, Bernard Kamsu-Foguem, Fana Tangara, Deep convolution neural network for image recognition, Ecological Informatics, Vol. 48, No. 11, 2018, pp. 257-268.

[12] Alice J. O'Toole, Carlos D. Castillo, Connor J. Parde, Matthew Q. Hill, Rama Chellappa, Face Space Representations in Deep Convolutional Neural Networks, Trends in Cognitive Sciences, Vol. 22, No. 9, 2018, pp. 794-809.

[13] Anthony G. Scanlan, Low power \& mobile hardware accelerators for deep convolutional neural networks, Integration, Vol. 65, 2019, pp. 110 127.

[14] Chih-Hsiao Tsai, Jung-Chuan Yen, The Development and Evaluation of a Kinect Sensor Assisted Learning System on the Spatial Visualization Skills, Procedia - Social and Behavioral Sciences, Vol. 103, No. 11, 2013, pp. 991-998.

[15] Zhen Yang, Cheng Chen, Yuqing Lin, Duming Wang, Weidan Xu, Effect of spatial enhancement technology on input through the keyboard in virtual reality environment, Applied Ergonomics, Vol. 78, No. 7, 2019, pp. $164-175$

[16] Kavisha Khanuja, Jaclyn Joki, Gloria Bachmann, Sara Cuccurullo, Gait and balance in the aging population: Fall prevention using innovation and technology, Maturitas, Vol. 110, No. 4, 2018, pp. 51-56.

[17] Yu Liang, Dalei Wu, Dakila Ledesma, Chris Davis, Zibin Guo, Virtual Tai-Chi System: A smart-connected modality for rehabilitation, Smart Health, Vol. 9-10, No. 12, 2018, pp. 232-249.

[18] Hassan Sadeghi, Mohammad Nazrul Hakim, Tengku Aizan Hamid, Saidon Bin Amri, Elham Shakoor, The effect of exergaming on knee proprioception in older men: A randomized controlled trial, Archives of Gerontology and Geriatrics, Vol. 69, No. 4, 2017, pp. 144-150.

[19] Maura Mengoni, Silvia Ceccacci, Andrea Generosi, Alma Leopardi, Spatial Augmented Reality: an application for human work in smart manufacturing environment, Procedia Manufacturing, Vol. 17, No. 1, 2018, pp. 476-483.

[20] Antonio Bernardo, The Changing Face of Technologically Integrated Neurosurgery: Today's High-Tech Operating Room, World Neurosurgery, Vol. 106, No. 10, 2017, pp. 1001-1014. 


\section{Biographies}

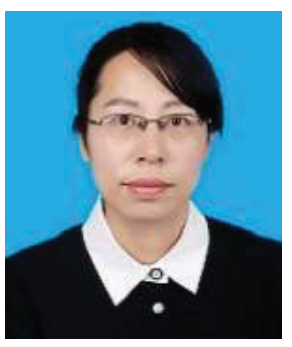

Yangbing Zheng, Associate Professor of control science and engineering, with Nanyang Normal University, Nanyang, China. She received her Bachelor of Engineering Science in Electronic Information Engineering from Nanyang Institute of Technology, Henan, China, in 2006; and the Doctor Degree of Engineering in detection technology and automatic equipment from China University of Mining and Technology, Beijing, China, in 2013, respectively. Her current research interests include active robot control, and nonlinear control.

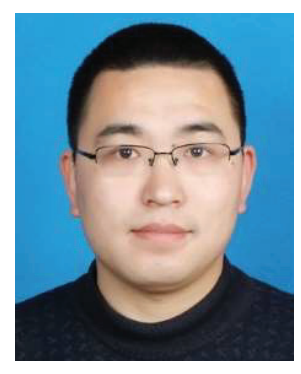

Xiao Xue, Associate Professor of School of Electronic and Electrical Engineering in Nanyang Institute of Technology, Nanyang, China. He received his Bachelor of Engineering Science in Electronic Information Engineering from Nanyang Institute of Technology, Henan, China, in 2003; the Doctor Degree of Engineering in detection technology and automatic equipment from China University of Geosciences, Wuhan, China, in 2015. His current research interests include Detection technology, and intelligent control. 


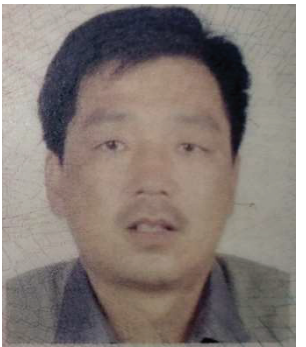

Jisong Zhang, President and Senior Engineer of Qinghai Wandong Ecological Environment Development Co.LTDzong. He has been engaged in the research of automatic control direction related to wolfberry planting. 
